\title{
ON CONTINUITY OF A PARTIAL ORDER
}

\author{
J. V. DESHPANDÉ 1
}

1. Introduction and summary. Let $(X, \leqq)$ be a poset; that is, $\leqq$ is a reflexive, antisymmetric and transitive relation on the set $X$. If $X$ is also a topological space, the usual way of defining the continuity of the partial order $\leqq$, is to require that its graph $P=\{(x, y): x \leqq y\}$ be closed in $X \times X$ (see, for example, [2], [3]). Ward [3], among others, has shown that the condition that $P$ be closed is equivalent to saying that if $a \$ b$, then there exist (open) neighborhoods $U_{a}$ and $U_{b}$ of $a$ and $b$ respectively such that $x \in U_{a}, y \in U_{b}$ implies that $x y$. This is, in fact, equivalent to saying that for $a, b \in X$, there exist (open) neighborhoods $U_{a}$ and $U_{b}$ such that (i) if $a<b$ then $x \in U_{a}$ and $y \in U_{b}$ implies that $y \leq x$ and (ii) if $a$ and $b$ are mutually incomparable, then $x \in U_{a}$ and $y \in U_{b}$ implies that $x$ and $y$ are mutually incomparable.

Here, another definition of the strong continuity of $\leqq$ is proposed and it is shown that (Theorem 2) for some semilattices, the strong continuity of the associated partial order insures that it is a topological semilattice, that is, the semilattice operation is continuous. One may note that this is not necessarily true if the partial order is continuous in the usual sense, even for a compact space.

All spaces are assumed to be Hausdorff. A neighborhood means an open neighborhood. A partial order may be denoted either by $\leqq$ or by its graph $P$. The closure of a set $A$ will be denoted by $A^{*}$, its interior by Int $A$ and its frontier by $F(A)$. The diagonal $\Delta$ of a set $X$ is the set $\{(x, x): x \in X\}$.

2. Strong continuity of a partial order. A partial order $P$ on a topological space $X$ is said to be strongly continuous if for every $a$, $b \in X$.

(i) if $a<b$, there exist neighborhoods $U_{a}$ and $U_{b}$ of $a$ and $b$, respectively, such that for every $x \in U_{a}$ and $y \in U_{b}, x<y$ holds.

(ii) if $a$ and $b$ are incomparable, there exist neighborhoods $U_{a}$ and $U_{b}$ of $a$ and $b$, respectively, such that every $x \in U_{a}$ is incomparable with every $y \in U_{b}$.

It is clear that a strongly continuous partial order is also continuous in the usual sense. The usual partial order on the Euclidean plane with the usual topology is continuous but not strongly so.

Presented to the Society, September 3, 1965; received by the editors October 3, 1966.

1 Present address: Center for Advanced Studies in Mathematics, University of Bombay, India. 
Theorem 1. For a partial order $P$ on a topological space $X$, the following are equivalent:

(1) $P$ is strongly continuous.

(2) $P=P^{*}$ and $P \backslash \Delta=\operatorname{Int}(P \backslash \Delta)$.

(3) $F(P) \subset \Delta$.

Proof. (1) $\Rightarrow(2)$. Since strong continuity of $P$ implies its continuity, we have that $P=P^{*}$. Let $(a, b) \in P \backslash \Delta$. Then by (i) of the definition of the strong continuity, there exist neighborhoods $U_{a}$ and $U_{b}$ of $a$ and $b$ respectively such that $U_{a} \times U_{b} \subset P \backslash \Delta$. Thus $(a, b) \in \operatorname{Int}(P \backslash \Delta)$. (2) $\Rightarrow(1)$. Since $P=P^{*}, P$ is continuous and part (ii) of the definition of the strong continuity holds. If $a<b$, then $(a, b) \in P \backslash \Delta$, which is open. Hence there exist neighborhoods $U_{a}$ and $U_{b}$ of $a$ and $b$ respectively such that $U_{a} \times U_{b} \subset P \backslash \Delta$. (2) $\Rightarrow(3)$. Since $P \backslash \Delta$ is open, $P \backslash \Delta$ $C$ Int $P$ and therefore $F(P) \cap(P \backslash \Delta)=\varnothing$. Since $P$ is closed, $F(P) \subset P$ $=(P \backslash \Delta) \cup \Delta$. Hence $F(P) \subset \Delta$. (3) $\Rightarrow(2)$. Since $F(P) \subset \Delta \subset P$, we have that $P=P^{*}$. Let $(a, b) \in P \backslash \Delta$. Then $(a, b) \in \operatorname{Int}(P)$ and there exist neighborhoods $U_{a}$ and $U_{b}$ of $a$ and $b$ respectively such that $U_{a} \times U_{b} \subset P$. Since $X$ is Hausdorff, $U_{a}$ and $U_{b}$ may be taken to be disjoint. Thus $U_{a} \times U_{b} \subset P \backslash \Delta$.

3. Partial order derived from a semilattice. Let $(X, \Lambda)$ be a semilattice, that is, let $\Lambda$ be a commutative, associative, idempotent operation on $X \times X$ to $X$. The relation $\leqq($ or $P$ ) on $X$ defined by $x \leqq y$ iff $x=x \Lambda y$ is a partial order [1]. The partial order $\leqq$ is order-dense if for every $x, y \in X, x<y$ implies the existence of $z \in X$ such that $x<z<y$.

THEOREM 2. Let $X$ be a compact, (Hausdorff) space with a semilattice operation $\Lambda$ and let $P$ be the associated partial order. If $X$ is order-dense in $P$, then the strong continuity of $P$ implies the continuity of $\Lambda$. The converse is not true.

Proof. Let $\left\{\left(x_{\alpha}, y_{\alpha}\right): \alpha \in A\right\}$ be a net in $X \times X$ converging to $(a, b)$. It suffices to show that the net $\left\{x_{\alpha} \Lambda y_{\alpha}: \alpha \in A\right\}$ converges to $a \Lambda b$

Case (i). Let $a<b$. Then $a=a \Lambda b$. Let $U$ be any neighborhood of $a=a \Lambda b$. By strong continuity of $P$, there exist neighborhoods $G_{a}^{\prime}$ and $G_{b}$ of $a$ and $b$ respectively such that $x \in G_{a}^{\prime}, y \in G_{b}$ implies $x<y$. Let $G_{a}=U \cap G_{a}^{\prime}$. Then $x \in G_{a}, y \in G_{b}$ implies $x \Lambda y=x \in G_{a} \subset U$.

Case (ii). Suppose $a$ and $b$ are mutually incomparable. Let $z=a \mathrm{~A} b$ and let $z^{\prime}$ be a cluster point of $\left\{x_{\alpha} \Lambda y_{\alpha}: \alpha \in A\right\}$.

Claim. $\boldsymbol{z}=\boldsymbol{z}^{\prime}$. 
One easily sees that $z^{\prime} \leqq z$. Now, if $z^{\prime}<z, z^{\prime}<z<a$ and $z^{\prime}<z<b$. Then, by repeated use of the definition of strong continuity of $P$, we can find neighborhoods $U, V, W_{1}$ and $W_{2}$ of $z^{\prime}, z, a$ and $b$ respectively such that for every $x \in U, y \in V, s \in W_{1}$ and $t \in W_{2}$ we have $x<y<s$ and $x<y<t$.

Since $x_{\alpha}$ converges to $a$ and $y_{\alpha}$ to $b ; x_{\alpha} \in W_{1}$ and $y_{\alpha} \in W_{2}$ whenever $\alpha \geqq \alpha_{0}$ for some $\alpha_{0}$. In other words for $\alpha \geqq \alpha_{0}, z<x$ and $z<y$ so that $z \leqq x_{\alpha} \Lambda y_{\alpha}$. But every $x \in U$ is such that $x<z$. Hence the net $\left\{x_{\alpha} \Lambda y_{\alpha}: \alpha \in A\right\}$ cannot be frequently in $U$, contradicting the fact that $z^{\prime}$ is its cluster point. Hence $z^{\prime}=z$.

Thus $z$ is the unique cluster point of $\left\{x_{\alpha} \Lambda y_{\alpha}: \alpha \in A\right\}$. Since $X$ is compact, this proves that $z$ is its unique limit point.

Case (iii): Let $a=b$. Again, let $z^{\prime}$ be a cluster point of $\left\{x_{\alpha} \Lambda y_{\alpha}: \alpha \in A\right\}$. By the same argument as in Case (ii) we see that $z^{\prime} \leqq a=a \Lambda b$. If $z^{\prime}<a$ then since $X$ is order-dense, there is $z \in X$ such that $z^{\prime}<z<a$. Arguing in the same way as in Case (ii), we see that in this case $z^{\prime}$ cannot be a cluster point of the net.

That the converse is not true is seen by considering the example of the unit square in the Euclidean plane with the usual partial order.

The condition that the space $X$ be order-dense cannot be dropped, as the following example shows.

EXAMPLE 1. Let $L$ be a horizontal straight line segment and with the usual Euclidean topology on it. Let $O$ be any point below $L$ (not on $L$ ) and with the discrete topology on it. Let $X=L \cup\{O\}$ and topologize $X$ by using as a base the union of topologies on $L$ and $O$. Define $\Lambda$ on $X \times X$ by

$$
\begin{aligned}
x \Lambda y=x & \text { if } x=y, \\
=0 & \text { if } x \neq y .
\end{aligned}
$$

Then $\Lambda$ is a semilattice operation on $X$ such that the associated partial order is strongly continuous. But $X$ is not order-dense and $\Lambda$ is not continuous.

Before closing, one may also note the semigroup version of Theorem 2 . It reads

THEOREM 3. Let $S$ be a compact, Hausdorff topological space on which is defined a commutative idempotent semigroup operation such that whenever $x \neq y$ and $x=x y$, there is $z \in S$ such that $x \neq z \neq y$ and $x=x z$ and $z=y z$. Let $P=\{(x, y): x=x y\}$. Then $F(P) \subset \Delta$ implies that $(S, \cdot)$ is a topological semigroup.

The author wishes to acknowledge with gratitude the help he 
received from Professor A. D. Wallace in the preparation of this article.

\section{REFERENCES}

1. G. Birkhoff, Lattice theory, 2nd ed, Colloq. Publ., Vol. 25, Amer. Math. Soc., Providence, R. I., 1948.

2. W. L. Strother, Continuous multi-valued functions, Bol. Soc. Mat. São Paulo 10 (1955), 87-120.

3. L. E. Ward, Partially ordered topological spaces, Proc. Amer. Math. Soc. 5 (1954), 144-161.

UNIVERSITY OF FLORIDA 